\title{
Comprehensive Architecture for Simulation of the Human Body based on Functional Anatomy
}

\author{
Sebastian Ullrich ${ }^{1}$, Jakob T. Valvoda ${ }^{1}$, Andreas Prescher ${ }^{2}$, Torsten Kuhlen ${ }^{1}$ \\ ${ }^{1}$ Virtual Reality Group, RWTH Aachen University \\ ${ }^{2}$ Institute for Neuroanatomy, University Hospital Aachen \\ Email: s.ullrich@rz.rwth-aachen.de
}

\begin{abstract}
In this paper we propose a structured approach for the simulation of the human body which is comprehensive and extendable. Our architecture resembles the human organism as defined by the systematic and functional anatomy to integrate a broad range of simulation algorithms. To share common data and to create interlinks between algorithms without modifying the algorithms themselves we introduce abstract control entities that mimic the basic setup of physiological systems. We utilize the model-view-controller pattern to establish a separation of algorithms and data. The structure was designed for the purpose of interactive simulation of the human body in virtual environments.
\end{abstract}

\section{Introduction}

The simulation of the human body is a challenging task that is still being broadly researched. Even though there are many simulators covering different medical areas, only few approaches are to a limited amount compatible between each other. Our goal is to create a framework to manage multiple algorithms for simulation of functional anatomy in order to create a virtual human patient that behaves realistically. The motivation is to provide an unified platform that allows to integrate already existing simulation algorithms and to prevent isolated solutions that are incompatible between each other. Ideally, the models and data sets of one approach should be interoperable and reusable for other approaches.

In order to achieve this goal, we adapt concepts from systematic and functional anatomy to software design. In systematic anatomy the human body is defined by a number of functional systems. The basic systems comprise the respiratory, cardiovascular, nervous, muscular, skeletal, and integumentary systems [1]. Further systems are digestive, excretory, endocrine, immune and reproductive. Each system fulfills a set of specific unique functions. These - often vital tasks are accomplished by organs and other structural anatomical components. On a macroscopic or abstract level the interdependencies and actions are described by functional physiology.

Because this taxonomy differentiates between functional (physiological) and structural (anatomical) components, it is well suited to derive a structure for simulation of the human organism. For this reasons we have chosen systematic and functional anatomy as a blueprint for the creation of a comprehensive system that is capable of extension and integration, as well as, adaptable to subjects. 
Fig. 1. Overview of the architecture for a virtual human organism, showing possible access mechanisms for simulation algorithms

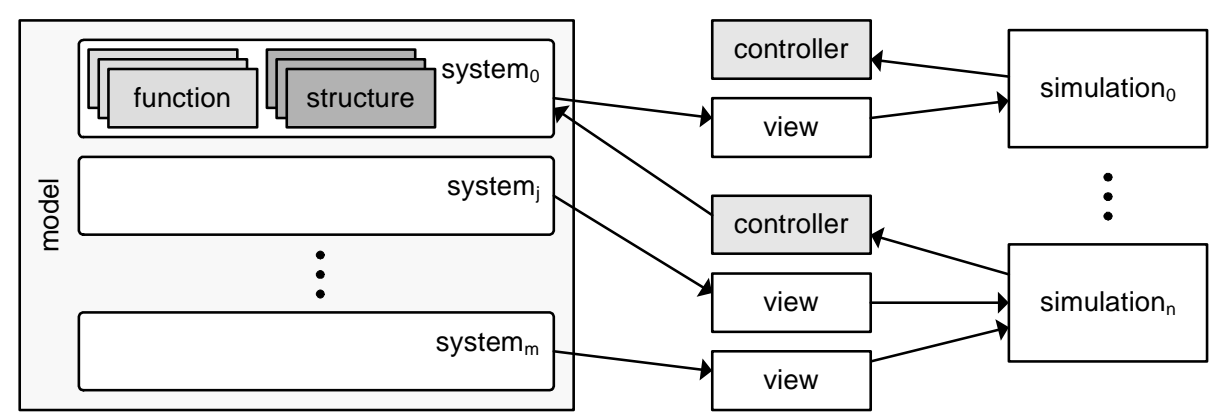

\section{State of the art and motivation}

Existing solutions are categorized by different fields of applications. Almost all surgical simulators focus on a set of procedures and implement simulations of restricted regions or specific layers of interest [2]. Current approaches for a unified simulation standard are still in early development. The international "Digital Human", consortium aims at combining micro- and macroscopic simulation [3]. Other examples are based on creating interactive anatomical atlases [4]. Cardenas et al. presented an extendible framework for medical image processing and visualization [5]. A comparable approach by Seifert et al. is used for surgical planning and also medical image processing [6]. The "'Digital Human"' project follows an intention similar to our approach, however, they have not proposed any system architecture yet. The other solutions presented above are restricted to one specimen (often the popular visible human data set) or to a limited amount of physiological systems. Until now, there is no systematic encapsulation of interindividually shared human anatomical and functional attributes.

Our structured approach allows to create comprehensive models of the human organism, based on functional systems, that strictly separates functionality from data. Thereby it enables individual, interoperable and reusable models that can be used by arbitrary simulation algorithms.

\section{Methods}

The architecture proposed in this paper consists of an abstract model for a human organism and defines access rules for simulation algorithms (Fig. 1). As mentioned before, we have adapted the distinction of functional systems and their inherent separation between physiology and anatomy, i.e., function and structure. Thus, the model consists of a set of functional systems. Furthermore, the data itself is stored in two types of interlinked containers: the functional and the structural component (Fig. 2). To provide access rules we use the model- 
Fig. 2. Partial setup of four systems. Additional components per system and many attributes are not shown here but are derived from systematic anatomy accordingly

\begin{tabular}{|c|c|c|c|c|c|}
\hline Integumentary & \begin{tabular}{c}
\multicolumn{2}{c}{ Nervous } \\
central $\quad$ peripheral
\end{tabular} & $\begin{array}{l}\text { Mus } \\
\text { skeletal }\end{array}$ & $\begin{array}{l}\text { ular } \\
\qquad \text { smooth }\end{array}$ & Skeletal & \\
\hline $\begin{array}{l}\text { Surface } \\
\text { skin->nerve } \\
\text { deform() } \\
\text { sense() }\end{array}$ & \begin{tabular}{l}
\multicolumn{1}{c}{ Cord } \\
nerve->muscle \\
innervate ()
\end{tabular} & $\begin{array}{l}\text { Polyline } \\
\text { Muscle->tend } \\
\text { actuate() }\end{array}$ & & $\begin{array}{l}\quad \text { Joint } \\
\text { tendon->bone } \\
\text { rotate() }\end{array}$ & $\begin{array}{c}\text { function } \\
\ldots .\end{array}$ \\
\hline$\downarrow$ & I & & I & $\downarrow$ & \\
\hline Border & i & $\begin{array}{l}\text { rol- } \\
\text { tt }\end{array}$ & & Segment & $\cdots$ \\
\hline Skin & $\begin{array}{c}\text { Nerve } \\
\text {-conductivity }\end{array}$ & $\begin{array}{c}\text { Muscle } \\
\text {-force/tonus }\end{array}$ & $\begin{array}{l}\text { Tendon } \\
\text {-stiffness }\end{array}$ & Bone & $\begin{array}{c}\text { structure } \\
\ldots . .\end{array}$ \\
\hline
\end{tabular}

view-controller pattern to separate data from algorithms. Subsequent sections describe the system, as well as its components.

\subsection{System Definition}

The physiological systems are based on their real-world counterparts. The key idea is that there are negligible differences of the purely functional aspects of a physiological system between individuals. In addition interrelations and links between components differ only morphologically but not on an abstract level, e.g., M. brachialis is always innervated by N. musculocutaneus. This abstraction allows to exchange underlying data sets (i.e. patient-specific scans, cp. figure 3) with only minor changes of some individual functional parameters.

The basic responsibility of a system, in our definition, is to establish logical links between functional and structural components and manage data access. In order to solve prioritization of access from simulation algorithms, and concurrency issues we propose the following schema. Every algorithm is allowed to access the resources one time only during each iteration. The requests are processed by a priority queue, whereby the priorities are set by the developer. We use dirt-flag containers to manage data update notification for simulation algorithms. This means that when a simulation algorithm initially requests a component it is automatically registered in the system. Thus, it can be later notified of changes through a view. After each simulation cycle every system iterates over its anatomical components and looks for changes. These changes induced by specific simulation controllers are distributed to all linked components and trigger the views of other simulations in the next cycle. 
Table 1. Example of a nerve stimulation procedure performed by two steps of user interaction and automatically following steps resolved by the respective systems (Fig. 2 ). The actions which are part of the functional components trigger attached simulation algorithms and thereby induce subsequent steps

\begin{tabular}{lllll}
\hline Nr Interaction & System & Action & Simulation algorithm \\
\hline 1 & needle insertion into shoulder & integumentary & deformation & FEM \\
2 & electric impulse from needle & nervous & stimulation & rule-based system \\
3 & actuation of innervated muscles & muscular & contraction & biomechanics \\
4 & pull of bone at insertion point & skeletal & rotation & kinematics \\
\hline
\end{tabular}

\subsection{Functional \& Structural Components}

Following the idea motivated in previous sections to encapsulate anatomical knowledge, the functional components define logical connections between structural components. Usually, a function is associated with two structural components the actuator and the receptor and a specific action.

Opposed to the functional components the structural components contain no logical connections and are patient specific. As a result, to simulate a new subject only the structural components need to be exchanged. They store raw data of arbitrary format (i.e. spatial information, physical attributes, geometric or volumetric representations, etc.).

\section{Results}

The structure proposed in this paper has been used in several scenarios. We present two applications, one focusing on interactive musculoskeletal simulation, and the other on regional anaesthesia. Both systems have been designed for the use in virtual environments.

The musculature application provides a tool-set for interactive modeling, simulation and representation of musculature. We have refactored an existing application with a layered approach [7] that utilizes two functional systems: the muscular and the skeletal system. Several biomechanical, kinematic and representational algorithms access these systems accordingly.

In addition to new functional systems the regional anaesthesia simulator shares both systems of the aforementioned application. The simulator is composed of functional systems (Fig. 2). Thereby, existing structures and also relevant algorithms have been reused. The update sequence during a virtual peripheral blocking procedure (Tab. 1) illustrates the interrelation between the particular functional systems.

\section{Discussion \& Conclusion}

We have presented a new data structure and architecture for simulation of virtual humans. Our approach is based on and motivated by systematic and functional anatomy. Even though there are various physiological systems with very 
Fig. 3. Dissectional ( $a$ ) and medical (b) imaging data are used within the components of systems (cp. figure 2) to form the base of a virtual simulated human body $(c)$
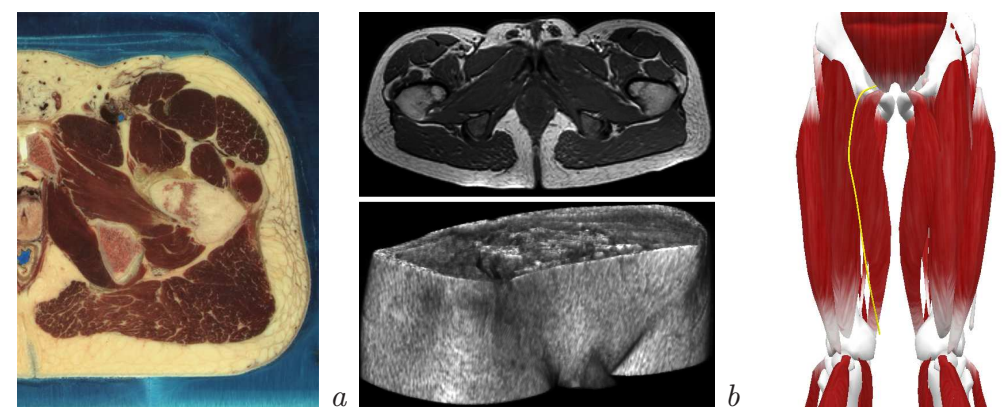

diverse functions we have established an abstract system base class with shared properties and common behavior for access management. Furthermore, we have provided a concept to allow for easy extension and creation of new systems by using functional and structural components.

\section{Acknowledgments}

This work was funded by the START-Programm (UK Aachen) and also supported by a grant from the German Research Foundation (DFG, KU 1132, LE 1108, RO 2000).

\section{References}

1. Leutert G, Schmidt W. Systematische und funktionelle Anatomie. 10th ed. Urban \& Fischer; 2004.

2. Liu A, Tendick F, Cleary K, Kaufmann C. A survey of surgical simulation: Applications, technology, and education. Presence 2003;12(6):599-614.

3. of American Scientists Federation. Digital Human Project. http://www.fas.org/dh/ index.html; 2006. Available from: http://www.fas.org/dh/index.html

4. Kriete A, Berger LC, Stallkamp J, Wapler M. Grundlagen eines interaktivfunktionellen Atlanten der menschlichen Anatomie. In: Procs BVM. Heidelberg; 1999. 253-257.

5. Cardenas S CE, Braun V, Hassenpug P, Thorn M, Hastenteufel M, Kunert T, et al. Ein Framework für die Implementierung von Anwendungssystemen zur Verarbeitung und Visualisierung von medizinischen Bildern. Procs BVM 2001; 142-146.

6. Seifert S, Kussaether R, Henrich W, Voelzow N, Dillmann R. Integrating Simulation Framework MEDIFRAME. In: IEEE EMBC. Cancun, Mexico; 2003. 1327-1330.

7. Valvoda JT, Ullrich S, Kuhlen T, Bischof $\mathrm{CH}$. Interactive biomechanical modeling and simulation of realistic human musculature in virtual environments. Procs BVM 2006; 404-408. 